\title{
Evaluation of the seismogenic (M6+) nodes for the territory of Bulgaria and adjacent areas
}

\section{Оценка на сеизмогенните (М6+) възли на територията на България и съседните райони}

\author{
Alexander Gorshkov ${ }^{1}$, Olga Novikova ${ }^{1}$, Sonia Dimitrova ${ }^{2}$, Aleksander Soloviev $^{1}$, Maxim Semka ${ }^{1}$, \\ Dragomir Dragomirov ${ }^{3}$ Milen Tsekov ${ }^{3}$ Lyuba Dimova ${ }^{3}$, Reneta Raykova ${ }^{3}$ \\ Александър Гориков ${ }^{1}$ Олга Новикова ${ }^{1}$, Соня Димитрова ${ }^{2}$, Александър Соловьов $^{1}$, \\ Максим Семка ${ }^{1}$, Драгомир Драгомиров ${ }^{3}$ Милен Цеков ${ }^{3}$, Люба Димова ${ }^{3}$ Ренета Райкова \\ ${ }^{1}$ Institute of Earthquake Prediction Theory and Mathematical Geophysics, RAS, Profsoyuznaya str., 84/32, 117997 Moscow; \\ E-mail: gorshkov@mitp.ru \\ ${ }^{2}$ National Institute of Geophysics, Geology and Geography, G. Bonchev blvd., bl. 3, 1113 Sofia; E-mail: syd@mail.bg \\ ${ }^{3}$ Sofia University St. Kliment Ohridski, Faculty of Physics, 5 J. Bourchier blvd., 1164 Sofia; E-mail: rraykova@phys.uni-sofia.bg
}

\begin{abstract}
In this study seismogenic nodes capable to generate earthquakes with magnitudes $M \geq 6$ are identified for the territory of Bulgaria and adjacent areas. Definition of nodes is based on a morphostructural zonation. Pattern recognition algorithm Cora-3 is applied to identify the seismogenic nodes, characterized by specific geological and geophysical data. The pattern recognition method is trained on information for 30 seismic events with $\mathrm{M} \geq 6$ for the period $29 \mathrm{BC}-2020$, selected from historical and instrumental Bulgarian earthquake catalogues. As a result, 56 seismogenic nodes are recognized, most of them in southwestern Bulgaria.
\end{abstract}

Keywords: morphostructural zonation, pattern recognition method, seismogenic nodes.

\section{Увод}

Територията на България не е толкова сеизмоактивна, колкото например близките Гърция или Италия, но това се отнася най-вече за честотата на земетресенията. Магнитудът на някои от най-силните български земетресения е съпоставим с този на най-силните земетресения в Европейската част на Алпо-Хималайския сеизмичен пояс. Основна роля в определянето на сеизмичната опасност в даден район играе именно точната информация за станалите земетресения, независимо каква методика за определяне на риска се използва. Целта на тази работа е да идентифицира и оцени зони с потенциал да генерират земетресения с магнитуд $\mathrm{M} \geq 6$.

Използваната методология се базира на хипотезата, че епицентрите на силните земетресения са разположени в морфоструктурни възли, определени посредством специфично морфоструктурно зониране и разпознати като сеизмогенни (Gelfand et al., 1972; Rantsman, 1979; Gorshkov et al., 2003). Дългогодишни изследвания в сеизмичноактивни зони показват голяма надеждност на методоло- гията $-86 \%$ от земетресенията, случили се след публикуване на подобно изследване за други райони, попадат в областта на възлите, разпознати като сеизмогенни (Soloviev et al., 2014; Gorshkov, Novikova, 2018).

Получената информация за разположението на възможни силни земетресения в сеизмогенните възли може да се използва директно в неодетерминистичните процедури за оценка на сеизмичната опасност (NDSHA), запълвайки възможни липси в наблюдаваната сеизмичност (Panza, Bela, 2020). По този начин са построени карти на сеизмичната опасност в няколко района (Peresan et al., 2011; Gorshkov et al., 2019).

\section{Методология}

Методологията за определяне на сеизмогенните възли се състои от две части: морфострукторно зониране (МС3) и метод на разпознаване (MP). В първата част се определят морфоструктурните възли, чрез използване на метода на морфострук- 
турно зониране. Целта на МС3 е да раздели района на йерархично подредени зони със сравнително еднородна топографска и тектонска структура на земната кора. По този начин се дефинират блокове от различен ранг (първи, втори и трети). Границите между отделните блокове дефинират морфоструктурните линеаменти. Пресечните точки на тези линеаменти дефинират възли. МС3 умишлено игнорира наблюдаваната сеизмичност. За всеки възел се определя набор от геолого-геофизични характеристики, разделени на 3 групи. В първата група се включват главно топографски и геоложки характеристики. Втората група включва различни параметри, определени от МС3, а третата група различни геофизични данни като Буге-аномалия и геомагнитна аномалия. Характеристиките на всеки възел определят неговия вектор. Във втората част, дефинираните от МС3 възли се класифицират с метода на разпознаване Cora-3 (Gelfand et al., 1972; Gorshkov et al., 2003). Използват се векторните характеристики на всеки възел. За да се „обучи“ процедурата се използват данни за наблюдаваните в района земетресения с магнитуд по-голям или равен на дадена прагова стойност $\mathrm{M}_{0}$. Всички възли, в чиято околност има наблюдавано такова земетресение, се дефинират от клас D. Процедурата търси възли с подобни характеристики и сред останалите такива. Крайният резултат включва две групи възли. Пьрвата група от възли са от клас D, в които могат да се генерират земетресения с магнитуд, надвишаващ дадената граница $\mathrm{M}_{0}$. Втората група включва възли от клас $\mathrm{N}$, в които могат да се генерират само земетресения с магнитуд по-мальк от $\mathrm{M}_{0}$. Стойността на граничния магнитуд $\mathrm{M}_{0}$ ce избира според сеизмичния режим в даден регион.

\section{Резултати}

Карта на МС3 на България е показана на фиг. 1. За съставянето на тази карта са използвани различни топографски, тектонски и геоложки карти, сателитни снимки, както и данни за геоморфологията, активните разломи и геологията на района (Dachev, 1988; Kotzev et al., 2001; Yosifov et al., 2020 и цитирани в тях източници). Районът на България е разделен на 7 блока от първи ранг и множество блокове от втори и трети ранг. Те дефинират 178 възела, като на всеки от възлите е присвоен вектор с геолого-геофизични характеристики. Източник на информация за различните параметри са карти в мащаб 1:500 000 от Националния геоложки фонд на Министерство на енергетиката в Република България (www.me.government.bg), както и базата данни GeoMapApp (www.geomapapp.org). Отделните характеристики се определят за област с радиус $25 \mathrm{~km}$ около даден възел.

За разделянето на възлите на класове $\mathrm{D}$ и $\mathrm{N}$ посредством софтуера за разпознаване Cora-3 ce използва информация за наблюдаваната сеизмичност (Grigorova et al., 1978; Solakov et al., 2020). Граничният магнитуд $\mathrm{M}_{0}$ е избран да бъде 6 , тъй като събития от такъв порядък се случват сравнително често като нанасят щети и причиняват жертви, които могат да бъдат значителни в гъстонасе-

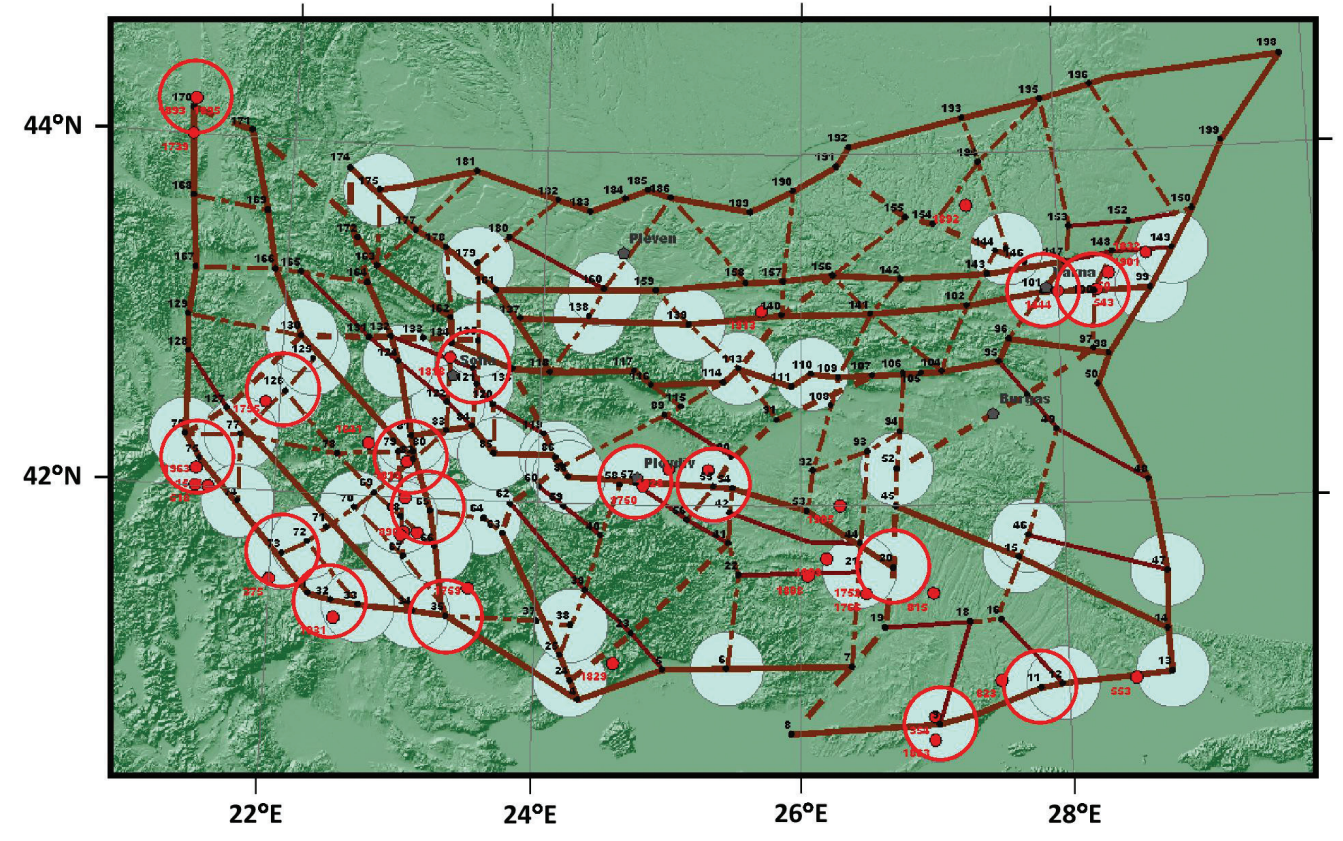

Фиг. 1. Морфоструктурна карта за района на България и разпознатите сеизмогенни възли, способни да генерират земетресения с магнитуд $\mathrm{M} \geq 6$. Линеаментите са показани с кафяви линии (дебелите - I ранг; средните - II ранг; тънките - III ранг; непрекъснатите - надлъжни; прекъснатите - напречни). Червените точки показват епицентрите на земетресения с $\mathrm{M} \geq 6$ и годината на съответното земетресение. Белите кръгове означават областта с радиус от $25 \mathrm{~km}$ около разпознатите сеизмогенни възли. Бели кръгове с червен контур означават възлите, асоциирани с наблюдаваната сеизмичност и използвани за „обучение“ на процедурата за разпознаване. С черни точки са означени останалите възли. 
лени райони. От използваните сеизмични каталози са избрани 30 събития с $M \geq 6$, които са асоциирани с 16 възела, дефинирани автоматично като тип $\mathrm{D}$ (показани на фиг. 1 като бели кръгчета с червен контур). Тези възли се използват от процедурата за разпознаване, за да идентифицират и други възможни възли с подобни характеристики. От всички 178 възела, 59 са разпознати като сеизмогенни, способни да генерират събития с магнитуд $\mathrm{M} \geq 6$ (показани на фиг. 1 с бели кръгчета).

Анализът на идентифицираните сеизмогенни възли показва, че с вече наблюдаваната сеизмичност освен първоначалните 16 „обучаващи“ възела, могат да се асоциират и други 20. Някои от тях могат да се асоциират с наблюдавана сеизмичност, която не е взета предвид при дефиницията на „обучаващите“ възли. Друга част от тези възли са близки, но не включват някои исторически земетресения, чието местоположение или магнитуд не са достатъчно прецизно определени.

Остават около 20 сеизмогенни възела, в чиято околност не е регистрирана сеизмична активност с $\mathrm{M} \geq 6$. Ако се допусне известна неточност в определянето на магнитудите и в анализа на тези сеизмогенни възли включим и земетресения с $\mathrm{M} \geq 5.8$, броят на „неактивните“ сеизмогенни възли ще намалее допълнително. Друга част от възлите потенциално могат да се свържат с разломна дейност в миналото, установена от геоложки изследвания. Остава една съществена част от сеизмогенни въ3ли, която не може да бъде свързана с никакви сеизмични прояви. Това е признак на възможна бъдеща сеизмична активност в близост до тези възли.

Трябва да се отбележи, че 3 от земетресенията през XX в. не са близо до сеизмогенен възел. Това може да се дължи на: скрити тектонски структури, пропуски в МСЗ или на неточно определен магнитуд на станалите земетресения.

\section{Заключение}

На територията на България са идентифицирани 59 възела, способни да генерират земетресения с магнитуд $\mathrm{M} \geq 6$. За целта са използвани морфоструктурно зониране и методология за разпознаване, тествани успешно върху сеизмична активност от различни части от земното кълбо. По-голямата част от сеизмогенните възли са локализирани в Югозападна България. Наблюдаваната сеизмичност (30 земетресения с $\mathrm{M} \geq 6$ ) е асоциирана с 16 от тези възли, използвани като еталон за разпознаване на сеизмогенни възли от използваната процедура. Има наблюдавана активност в близост до 20 от разпознатите като сеизмогенни възли, но в близост до други 20 такава активност не е наблюдавана. Към част от тези възли може да се асоциира земетръсна активност с магнитуд $\mathrm{M} \geq 5.8$, като се има предвид неточността в определянето на магнитуда на земетресенията, но остават значителен брой разпознати сеизмогенни възли, около които не е документирано никакво сеизмично събитие. Тази информация може да запълни евентуални пропуски в идентифи- цирани, на базата на сеизмичния каталог на България, активни сеизмогенни зони, и така се подобрява моделът на сеизмогенните източници. Получените резултати може да се използват в съставянето на дългопериодни карти на сеизмичната опасност, както и да бъдат използвани при неодетерминистична оценка на сеизмичния риск.

Благодарности: Това изследване е финансирано частично от Руския Фонд за Фундаментални Изследвания (договор № 20-55-18008) и от Фонд Научни Изследвания на МОН (договор № КП06-Русия/29 от 2020 г.).

\section{Литература \\ References}

Dachev, Ch. 1988. Structure of the Earth's Crust in Bulgaria. Sofia, Technika, 364 p. (in Bulgarian).

Gelfand, I., Sh. Guberman, M. Izvekova, V. Keilis-Borok, E. Ranzma. 1972. Criteria of high seismicity determined by pattern recognition. - Tectonophys., 13, 415-422; https:// doi.org/10.1016/0040-1951(72)90031-5.

Gorshkov, A., O. Novikova. 2018. Estimating the validity of the recognition results of earthquake prone areas using the ArcMap. - Acta Geophys., 66, 5, 843-853; https://doi. org/10.1007/s11600-018-0177.

Gorshkov, A., V. Kossobokov, A. Soloviev. 2003. Recognition of earthquake prone areas. - In: Keilis-Bork, V., A. Soloviev (Eds.). Nonlinear Dynamics of the Lithosphere and Earthquake. Heidelberg, Prediction, Springer, 235-320.

Gorshkov, A., H. Hassan, O. Novikova. 2019. Seismogenic nodes $(M \geq 5.0)$ in north-east of Egypt and implication for seismic hazard assessment. - Pure Appl. Geophys., 176, 2, 593-610; https://doi.org/10.1007/s00024-018-2012-9.

Grigorova, E., L. Christoskov, D. Sokerova, S. Rizhikova, A. Roglinov. 1978. Catalogue of earthquakes in Bulgaria and the nearby territories during the period 1st cent. BC - 1977. Archives Bull. Inst. Geophys., Bulg. Acad. Sci. (in Bulgarian).

Kotzev, V., R. Nakov, B. Burchfiel, R. King, R. Reilinger. 2001. GPS study of active tectonics in Bulgaria: results from 1996 to 1998. - J. Geodynam., 31, 189-200; https:// doi.org/10.1016/S0264-3707(00)00027-2.

Panza, G., J. Bela. 2020. NDSHA: A new paradigm for reliable seismic hazard assessment. - Engineer. Geol., 275, 105403; https://doi.org/10.1016/j.enggeo.2019.105403.

Peresan, A., E. Zuccolo, F. Vaccari, A. Gorshkov, G. Panza. 2011. Neo-deterministic seismic hazard and pattern recognition techniques: Time-dependent scenarios for NorthEastern Italy. - Pure Appl. Geophys., 168, 583-607; https:// doi.org/10.1007/s00024-010-0166-1.

Rantsman, E. 1979. Morphostructure of Mountain Regions and Sites of Earthquakes. Nauka, Moscow (in Russian).

Solakov, D., S. Simeonova, P1. Raykova, I. Aleksandrova. 2020. Catalogue of the earthquakes in Bulgaria and surroundings since 1981. - National Institute of Geophysics, Geodesy and Geography, BAS; https://doi.org/10.34975/ ctlg-2020.v.1.

Soloviev, A., A. Gvishiani, A. Gorshkov, M. Dobrovolsky, O. Novikova. 2014. Recognition of earthquake-prone areas: Methodology and analysis of the results. - Izv., Phys. Solid Earth, 50, 151-168; https://doi.org/10.1134/ S1069351314020116.

Yosifov, D., R. Radichev, R. Raykova. 2020. Geophysics of the Lithosphere and Nonlinear Metallogeny of Bulgaria. - MGU Publishing House "St. Ivan Rilski", 278 p. (in Bulgarian). 